\title{
Clinical Efficacy of Nimodipine and Edaravone Combination in the Treatment of Acute Cerebral Hemorrhage
}

\author{
SUZHEN WANG*, XIAOQIN AN¹, YUEHUA WANG, JIXIN ZHANG, LIGUO QI² \\ Department of Neurosurgery, ${ }^{1}$ Department of Neurology, Shandong Jiyang Public Hospital, Jinan 251400, China, \\ 2Department of Neurosurgery, Taian Central Hospital, Taian 271000, China
}

Wang et al: Efficacy of nimodipine and edaravone combination in acute cerebral hemorrhage

\begin{abstract}
To observe and analyze the clinical efficacy of nimodipine and edaravone combination in the treatment of acute cerebral hemorrhage, a total of 180 patients definitely diagnosed as acute cerebral hemorrhage and treated in Shandong Jiyang Public hospital were enrolled. The patients were divided into the control group and the research group with 90 patients in each group. Of those, patients of the control group were treated with edaravone, while a combination of nimodipine and edaravone was administered to patients in the research group. Therapeutic efficacy, neurologic impairment score, life quality score, hematoma volume and edema volume were compared between both the groups. Results obtained showed that the total therapeutic efficacy of the research group was better than that of the control group $(P<0.05)$. Measurement of patients' hematoma volume and edema volume before and after treatment indicated that the research group after drug administration demonstrated significant improvement over the control group $(\mathbf{P}<0.05)$. Moreover, there was a greater decrease in the neurologic impairment score of the research group than that of the control group $(\mathbf{P}<\mathbf{0 . 0 5})$. Combination of nimodipine and edaravone in the treatment of acute cerebral hemorrhage achieved better outcomes indicating greater therapeutic efficacy.
\end{abstract}

Key words: Nimodipine, edaravone; drug combination, acute cerebral hemorrhage, clinical efficacy

Cerebral hemorrhage is a common severe disease in the neurology department with a relatively high incidence. The patient's neurological function would be damaged to a various degree when acute cerebral hemorrhage occurred, causing severe impacts on patients'life quality or even threatening their lives ${ }^{[1,2]}$. With continuing clinical research, drugs for the treatment of cerebral hemorrhage have been identified including edaravone, nimodipine and some others, which became a ray of hope for those suffering from cerebral hemorrhage ${ }^{[3,4]}$. The key for improving the therapeutic efficacy lies in active and effective treatment regimens. Acute cerebral hemorrhage (fig. 1) refers to bleeding caused by nontraumatic vessel rupture in the brain parenchyma, for which drug treatment is usually applied. As a powerful hydroxyl radical scavenger, edaravone could penetrate the brain-blood barrier with high efficiency as well as protect neurons and promote their recovery. Moreover, nimodipine is a calcium channel antagonist, which could effectively dilate the brain blood vessels ${ }^{[5]}$ as well as reduce vasoconstriction, thus relieving vasospasm and improving hemoperfusion of the brain. This study was undertaken to observe and analyze the efficacy of the combination of nimodipine and edaravone in the treatment of acute cerebral hemorrhage. A total of 180 acute cerebral hemorrhage patients treated at the Shandong Jiyang Public Hospital from January 2016 to August 2018 were enrolled. All cases were in line with the diagnostic criteria of acute cerebral hemorrhage advanced by The Fourth Cerebrovascular Diseases Conference. Meanwhile, head CT or MRI

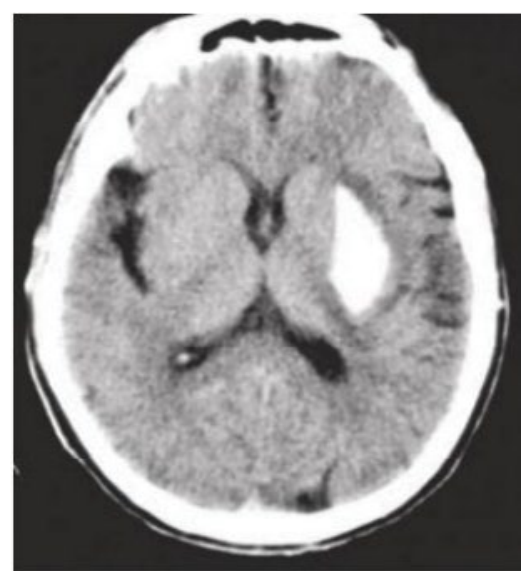

Fig. 1: The image of acute cerebral hemorrhage 
test were adopted to confirm diagnosis (fig. 2). The inclusion criteria were as follows ${ }^{[6]}$, the onset of cerebral hemorrhage was within $24 \mathrm{~h}$, complicated with limb paralysis and the muscle strength was less than level IV; the patients and their family enjoyed the right to information and signed a formal consent form; cerebral hemorrhage should not be caused by tumor, brain trauma, coagulation disorders and the like; there were no complications like infectious diseases and organ failure; no patient to be detected with a malignant tumor or allergic to the medication used in this study. This research was approved by the ethics committee of Shandong Jiyang Public Hospital. Patients were randomized into the research group and the control group, with 90 patients in each group. Of those, there were 50 males and 40 female in the research group with an average age of $70.68 \pm 3.25 \mathrm{y}$. There were 46 males and 44 females in the control group with an average age of $71.29 \pm 4.02 \mathrm{y}$. Data obtained from both groups in terms of sex, age, degree of education and family situation were all found to be comparable.

Routine therapeutic approaches included in-time dehydration to lower the intracranial pressure, oxygen uptake, lipid metabolism mediation, maintenance treatment of water electrolyte balance and acidbase balance, as well as prevention and treatment of complications. Edaravone was given through intravenous drip, $30 \mathrm{mg}$ of edaravone injection (Simcere Pharmaceutical Group, H20050280) was added into $100 \mathrm{ml}$ of sodium chloride injection $(0.9 \%)$ for

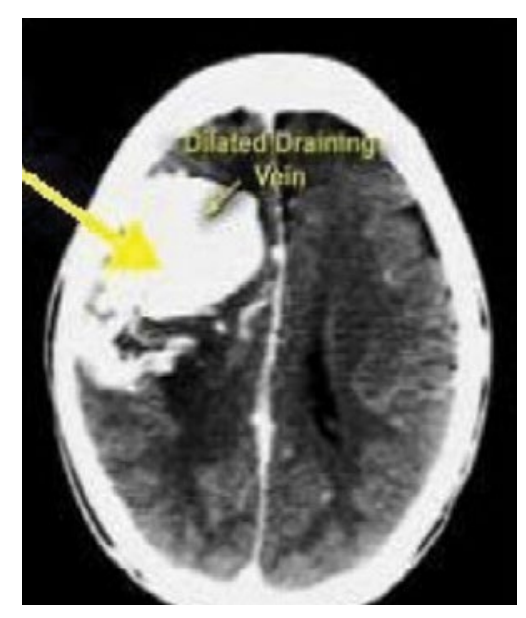

Fig. 2: The image of a case of acute cerebral hemorrhage intravenous drip. Edaravone was administered twice a day, for a consecutive month. In similar lines, a combination of nimodipine and edaravone was applied to the research group. The therapeutic regimen of edaravone was the same as the control group, but additionally $10 \mathrm{mg}$ of nimodipine (Bayer Company, J20100002) was added into $100 \mathrm{ml}$ of glucose injection $(5 \%)$ for intravenous drip, once a day. After 10 consecutive days of treatment, intravenous drip of nimodipine was changed into oral administration of nimodipine tablet (Bayer Company, H20003010), $60 \mathrm{mg}$ each time, 3 times a day. Similarly, the treatment was also given for a month. The total clinical efficacy of both groups were observed and compared, which included four standards, namely basic recovery, excellent, valid and invalid. Of those, the total clinical efficacy was evaluated according to the National Institute of Health stroke scale (NIHSS) [7]. Basic recovery refers to a decline of $91-100 \%$ in NIHSS after $2 \mathrm{w}$ of treatment, while excellent refers to a decline of $50-91 \%$ in NIHSS. If the NIHSS decreases by $21-50 \%$, then it is considered valid. If the NIHSS decreased less than $20 \%$ or the condition is even worse, then it is considered invalid. Moreover, the NIHSS, Activity of daily living (ADL) score, hematoma volume and edema volume were recorded. Statistical analysis was performed using SPSS21.0. All quantitative data were expressed in the form of mean \pm standard deviation, and comparisons were made using the t-test. Enumeration data were expressed in the form of natural number $(\mathrm{n})+$ percentage $(\%)$, and comparisons were made with chi-square test. $P<0.05$ represents the intergroup difference was of statistically significance. As shown in Table 1, the total clinical efficacy of the research group was significantly higher than that of the control group $(P<0.05)$. The images of a patient before and after treatment were shown in fig. 3. As shown in Table 2, no significant difference was observed in the hematoma volume and edema volume before treatment between both groups. After adopting different therapeutic regimens, the improvement in the research group was significantly greater than that of the control group $(P<0.05)$. As shown in Table 3, no significant difference was observed regarding the NIHSS score and ADL score before treatment between

TABLE 1: COMPARISON OF THE TOTAL CLINICAL EFFICACY BETWEEN BOTH GROUPS

\begin{tabular}{|c|c|c|c|c|c|}
\hline Groups & Basic recovery & Excellent & Valid & Invalid & Total clinical efficacy \\
\hline Research group $(n=90)$ & 30 & 46 & 10 & 4 & $86(95.56)$ \\
\hline Control group $(n=90)$ & 14 & 32 & 28 & 16 & $74(82.22)$ \\
\hline$X^{2}$ & & & & & 12.59 \\
\hline$P$ & & & & & $<0.05$ \\
\hline
\end{tabular}


both groups. After treatment, the scores of these 2 indicators of the research group were significantly better than that of the control group $(\mathrm{P}<0.05)$.

Studies previously reported showed that cerebral hemorrhage accounts for $20-30 \%$ of all stroke cases and the mortality rate in the acute phase is $30-40 \%{ }^{[8]}$. The causes of cerebral hemorrhage are mainly related to the pathological changes in the cerebrovascular system, that is, closely related to hyperlipemia, diabetes, hypertension, aging of blood vessels, smoking and the like. Patients suffered from cerebral hemorrhage often have sudden onset due to emotional agitation or strenuous exertion. The mortality is rather high in the early stage. Most survivors suffered from different degrees of dyskinesia, cognitive impairment, speech dysphagia and other sequelae. In the acute cerebral hemorrhage, most of the channel proteins are deactivated, which changes the permeability of the cell

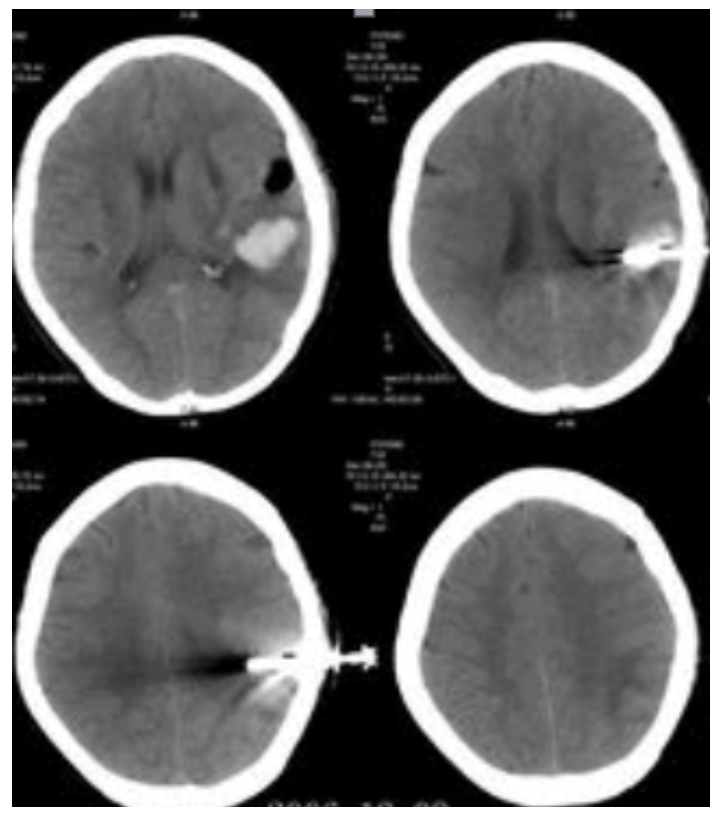

Fig. 3: Images of a patient before and after treatment membrane. Moreover, cellular apoptosis occurs and causes damage to the brain tissue. During the treatment of acute cerebral hemorrhage, on one hand, it is necessary to effectively stabilize the hemorrhagic foci and on the other hand, it is necessary to actively control the secondary pathologic changes such as cerebral edema, limiting its further development. As a typical neuroprotective agent, edaravone has small molecular weight as well as high lipophilicity. If the oxygen free radicals were neutralized at the same time, it is possible to significantly relieve the inflammatory response in the brain tissue as well as prevent and avoid neuronal apoptosis there by actively improving ischemia and hypoxia as well as promoting functional recovery of the neurons. Nimodipine could block the calcium channel on the cell membrane and inhibit the transfer of calcium into cell under hypoxia, thus lowering the cellular calcium concentration. Meanwhile, nimodipine could penetrate through the brain blood barrier and could exert favorable neuron-protective effect. Furthermore, nimodipine could actively alleviate the vasospasm of brain vessels under hypoxia, thus promoting the microcirculation in the point of bleeding and improving both oxygen and blood supply. Results from this study indicated that the total therapeutic efficacy of the research group was significantly better than that of the control group $(P<0.05)$. By measuring patients' hematoma and edema volume before and after treatment, it became possible to show that the improvement in the research group after drug administration was significantly greater than that of the control group $(P<0.05)$. Moreover, there was a greater reduction in the neurologic impairment score of the research group than that of the control group $(P<0.05)$. These findings are in line with relevant reports ${ }^{[9-16]}$. To sum up, treatment with a combination of nimodipine and edaravone for acute cerebral hemorrhage could

TABLE 2: HEMATOMA AND EDEMA VOLUME OF BOTH GROUPS BEFORE AND AFTER TREATMENT

\begin{tabular}{lcccc}
\hline \multirow{2}{*}{ Groups } & \multicolumn{2}{c}{ Hematoma volume $(\mathrm{ml})$} & \multicolumn{2}{c}{ Edema volume $(\mathrm{ml})$} \\
\cline { 2 - 5 } & Before treatment & After treatment & Before treatment & After treatment \\
\hline Research group $(\mathrm{n}=90)$ & $20.50 \pm 3.32$ & $9.70 \pm 2.29$ & $6.56 \pm 2.53$ & $2.53 \pm 4.19$ \\
Control group $(\mathrm{n}=90)$ & $21.66 \pm 3.20$ & $16.55 \pm 1.98$ & $6.19 \pm 2.78$ & $4.60 \pm 3.92$ \\
$\mathrm{t}$ & 0.34 & 8.49 & 1.02 & 13.59 \\
$\mathrm{P}$ & $>0.05$ & $<0.05$ & $>0.05$ & $<0.05$ \\
\hline
\end{tabular}

TABLE 3: THE NIHSS AND ADL SCORES OF BOTH GROUPS BEFORE AND AFTER TREATMENT

\begin{tabular}{lcccc}
\hline \multirow{2}{*}{ Groups } & \multicolumn{2}{c}{ NIHSS score } & \multicolumn{2}{c}{ ADL score } \\
\cline { 2 - 5 } & Before treatment & After treatment & Before treatment & After treatment \\
\hline Research group $(\mathrm{n}=90)$ & $23.50 \pm 3.32$ & $11.70 \pm 2.29$ & $32.56 \pm 2.53$ & $55.83 \pm 4.19$ \\
Control group $(\mathrm{n}=90)$ & $23.66 \pm 3.20$ & $16.55 \pm 1.98$ & $32.19 \pm 2.78$ & $45.60 \pm 3.92$ \\
$\mathrm{t}$ & 0.34 & 8.49 & 1.02 & 13.59 \\
$\mathrm{P}$ & $>0.05$ & $<0.05$ & $>0.05$ & $<0.05$ \\
\hline
\end{tabular}


achieve good clinical efficacy. Edaravone could improve the permeability of the brain blood barrier, actively promote the removal of free radicals and alleviate the damage to cells and neurons. Nimodipine due to its liposolubility could actively alleviate the vasospasm of brain vessels. Moreover, nimodipine could effectively improve the brain circulation, decrease the intracranial hematoma and promote hematoma absorption. With the combination of these two drugs, the volume of hematoma and edema as well as the NIHSS score were significantly decreased, thus the total therapeutic efficacy was significantly improved. This drug combination therapy is an important measure to improve life quality, with improved safety and acceptability. Therefore, nimodipine and edaravone combination treatment for acute cerebral hemorrhage could bring better outcomes, which is worth clinical promotion.

\section{REFERENCES}

1. $\mathrm{Yu}$ JH. Clinical efficacy of Nimodipine combined with Edaravone in the treatment of 56 patients with acute cerebral hemorrhage. Chinese J Mod Drug Appl 2016;10(22):143-4.

2. Li HR. Protective effect of nimodipine on PC12 cell apoptosis induced by $\mathrm{H} 2 \mathrm{O} 2$. Chinese J Comparative Med 2017;27(04):14-9.

3. Chen Y. Comparative observation of nimodipine and edaravone in the treatment of ischemic brain injury after intracerebral hemorrhage. Xinxiang Medical School, 2016.

4. Richard AB, Christopher JM, Philip DB, Alexander DS, James MM, Christopher PG. Risk stratification for ST segment elevation myocardial infarction in the era of primary percutaneous coronary intervention. World J Cardiol 2014; 6(08):865-72.

5. Hua Z, He XY, Zhuang SW, Wang J, Lai Y, Qi WG, et al. Clinical and procedural predictors of no-ref low in patients with acute myocardial infarction after primary percutaneous coronary intervention. World J Emerg Med, 2016, 5(02):96102.

6. Wang YQ, Xu J, Guo R, Xu CX, Hao YM, Chen CF, et al. Therapeutic effect in patients with coronary heart disease based on information analysis from Traditional Chinese Medicine four diagnostic methods. J Tradit Chin Med, 2017, 34(01):34-41.
7. Si KY, Li ZP. Effect of edaravone combined with nimodipine on the biochemical indexes of acute large area cerebral infarction. Chinese J Practical Nerv Dis 2017; 20(19):71-3.

8. Lv JL, Arsen A. Clinical efficacy of edaravone combined with nimodipine in the treatment of hypertensive intracerebral hemorrhage. J Clin Med Res Literature 2018;5(02):143-4.

9. Zhang M, He HW, Wang ZM, Xu ZH, Zhou NT, Tao ZX, et al. Diagnostic and prognostic value of minor elevated cardiac troponin levels for percutaneous coronary intervention-related myocardial injury: a prospective, single-center and doubleblind study. J Biome Res 2014; 28(02):98-107.

10. Li RJ, Hao PP, Chen YG, Zhang Y. Association of cystatin C level and cardiovascular prognosis for patients with preexisting coronary heart disease: A meta-analysis. Chinese Sci Bull 2014;59(Z1):539-45.

11. Demirel B, Bicakci U, Rizalar R, Alpaslan PF, Aydin O. Histopathological effects of mesenchymal stem cells in rats with bladder and posterior urethral injuries. Turk J Med Sci 2017; 47(6):1912-19.

12. Gao W, Wang Y, Basavanagoud B, Muhammadkamran J. Characteristics studies of molecular structures in drugs. Saudi Pharm J 2017; 25(4): 580-6.

13. Parisi L, Salerno M, Maltese A, Tripi G, Romano P, Di Folco A, et al. Paternal shift-working and sleep disorders in children affected by primary nocturnal enuresis. Acta Medica Mediterr 2017; 33(3): 481-4.

14. Sadi B, Bayat M, Tajik P, Hashemi S. Citrinin detection by intensified fluorescence signal of a fret-based immunosensor using magnetic/silica core-shell. Saudi J Biol Sci 2018; 25(1):171-7.

15. Saheed S, Taofik S, Oladipo A, Tom A. Spondias mombin 1. (Anacardiaceae) enhances detoxification of hepatic and macromolecular oxidants in acetaminophen-intoxicated rats. Pak J Pharm Sci 2017;30(6): 2109-17.

16. Yadav KD, Chaudhary AK, Verma AK. Bioavailability enhancement of partially water soluble solid medicament in traditional system of medicine. Indian J Pharm Sci 2017;79(5): 667-73.

This is an open access article distributed under the terms of the Creative Commons Attribution-NonCommercial-ShareAlike 3.0 License, which allows others to remix, tweak, and build upon the work non-commercially, as long as the author is credited and the new creations are licensed under the identical terms

This article was originally published in a special
issue: Special issue on "Animal Models \& Experimental
Medicine"
Indian J Pharm Sci 2020:82(1)spl issue4;64-67

